\title{
Cavernous Hemangioma in Cranial Vault: A Case Report
}

\author{
Jong-Seon Park, Sung-Kyun Hwang \\ Seoul, Republic of Korea \\ Corresponding author: \\ Sung-Kyun Hwang \\ Department of Neurosurgery, Ewha Womans \\ University Mokdong Hospital, Ewha Womans \\ University College of Medicine, 1071, \\ Anyangcheon-ro, Yangcheon-gu, Seoul 07985, \\ Republic of Korea \\ Tel: $+82-2-2650-2872$ \\ Fax: $+82-2-2650-2652$ \\ E-mail: nshsg@ewha.ac.kr
}

Department of Neurosurgery, Ewha Womans University Mokdong Hospital, Ewha Womans University College of Medicine,

Received: March 5, 2020

Revised: April 9, 2020

Accepted: April 11, 2020
Cavernous hemangioma $(\mathrm{CH})$ in cranial vault is rare comprising about less than $1 \%$ of all benign neoplasms of the bone. The vertebral column is most often affected, followed by the cranial vault. We describe a case of $\mathrm{CH}$ in parietal cranial vault. $\mathrm{A}$ 6-year-old child presented with skull depression. Brain computed tomography scan revealed a bone defect due to extrinsic compression by soft tissue mass at right parietal bone. This lesion was not without intracranial extension or associated brain abnormality. Brain magnetic resonance image showed a lesion of signal abnormality with low signal intensity on T1 image and high signal intensity on T2-weighted images with no enhancement. She was treated by operation with total excision of mass followed by a cranioplasty. Pathologic examination revealed a capillary hemangioma consisting of thin-walled blood vessels, some of which were distended with blood. She recovered to a favorable state without any neurological deficit.

Key Words: Child; Hemangioma, cavernous; Skull

\section{INTRODUCTION}

Primary cavernous hemangiomas $(\mathrm{CH})$ in cranial vault were uncommon and rare tumor, accounting for less than $1.0 \%$ of all bone neoplasms. These tumors are mostly found in vertebral bodies $^{1-3,5,6,8-10)}$. We report a case of $\mathrm{CH}$ in parietal cranial vault and successfully managed with surgical resection.

\section{CASE REPORT}

A 6-year-old right-handed female child presented with skull depression which was detected by her mother. She reported previous intermittent episodes of headaches within the past month. She had no significant medical or surgical history and her general and physical examination was normal. An initial work-up of the patient included a brain computed tomography (CT) scan, which revealed a ovoid bone defect due to extrinsic compression by soft tissue mass at right parietal bone $(1.0 \times$ $0.4 \mathrm{~cm}$; Fig. 1). This lesion was not without intracranial extension or associated brain abnormality. Possible preoperative diagnosis was interpreted as an underlying epidermal inclusion cyst or small low flow vascular malformation such as lymphangioma. Brain magnetic resonance image (MRI) showed a lesion of signal abnormality on the right parietal bone with indentation to adjacent bone. On unenhanced T1 image, this area exhibited low signal intensity, and high signal intensity on the T2-weighted images with no enhancement on the post contrast images. On the coronal images, there was no intracranial extension or associated brain abnormality. Note was made of erosion of the overlying cranial vault (Fig. 2).

She was treated by operation with total excision of mass followed by a cranioplasty. At operation, abnormal soft tissue was noted in the right parietal bone defect with the presence of hemorrhagic tissue (Fig. 3A). The lesion and bone were removed all the way along with $0.5 \mathrm{~cm}$ of normal bone margin. Pathologic examination of the lesion and bone revealed a capillary hemangioma consisting of thin-walled blood vessels, some of which were distended with blood. The hemangioma invaded the bone which showed some reactive osteoblastic
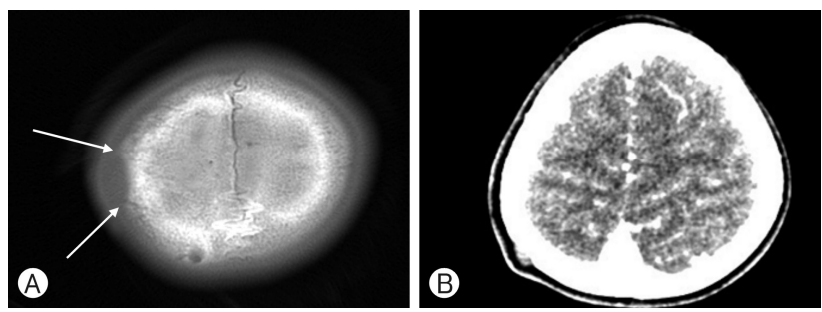

Fig. 1. (A, B) Brain computed tomography axial image demonstrating a ovoid bone defect due to extrinsic compression by soft tissue mass at right parietal bone $(1.0 \times 0.4 \mathrm{~cm})$. This lesion was not without intracranial extension or associated brain abnormality. 

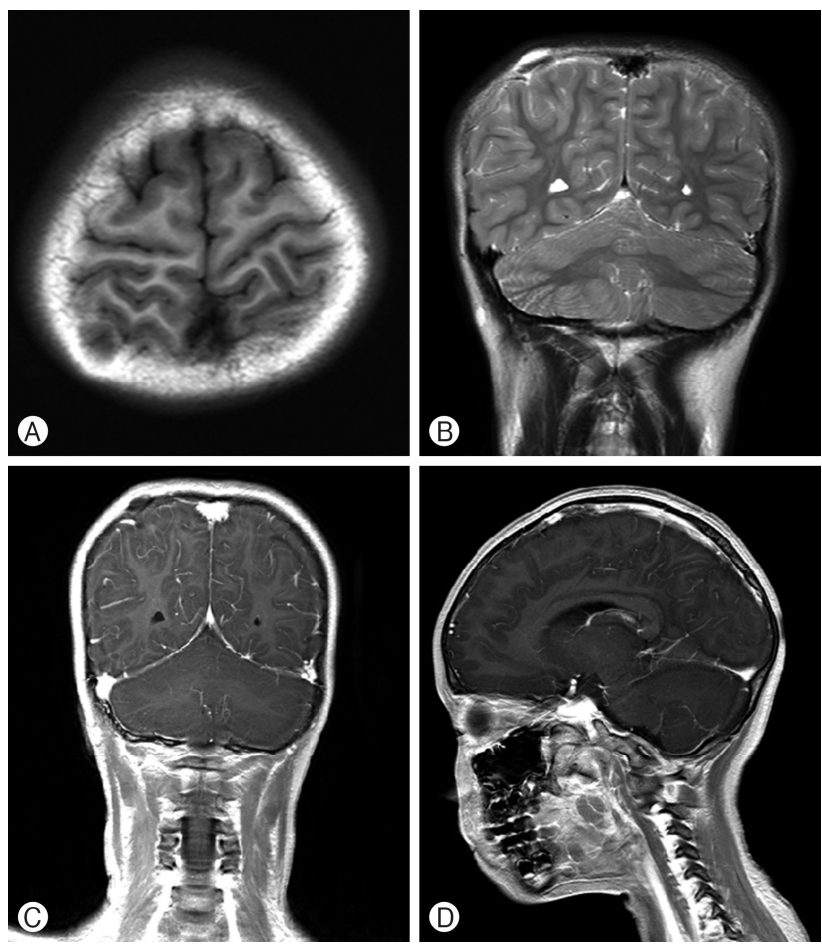

Fig. 2. (A, B) Brain magnetic resonance imaging $T 1$ and $T 2$ weighted image demonstrated the lesion of signal abnormality with low signal intensity on T1 and low signal intensity on T2. (C, D) Enhanced T1 coronal and sagittal image showed no enhancement with erosion of the overlying cranial vault.

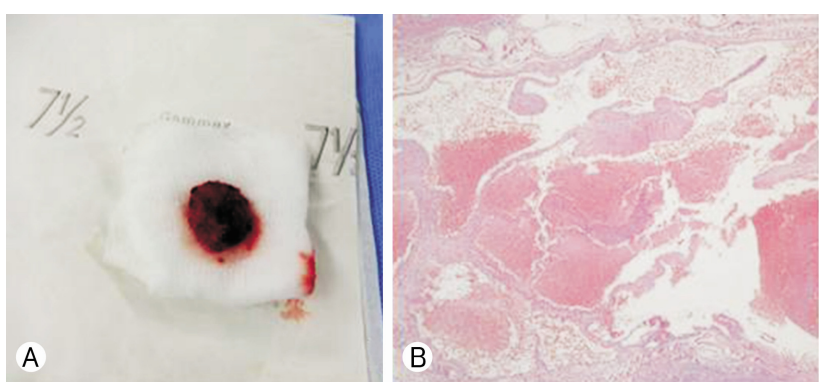

Fig. 3. (A) Operative finding, abnormal soft tissue was noted in the bone defect with the presence of hemorrhagic tissue. (B) Pathological finding (hematoxylin and eosin stain [H\&E], $\times 40$ magnification). It showed the typical appearance of cavernous hemangioma of honeycomb network of vascular spaces lined with endothelial cells and sparse amounts of loose connective tissue.

activity with new bone formation (Fig. 3B). She recovered to a favorable state without any neurological deficit.

\section{DISCUSSION}

$\mathrm{CH}$ in cranial vault, are rare benign tumors of blood vessels, more commonly found in the spinal vertebral column.
Overall, they represent less than $1 \%$ of all osseous benign tumors $^{1-3,5,6,8-10)}$. The parietal bone is thought to be the most commonly affected, followed by the frontal bone and less frequently by occipital and temporal bones. They are usually solitary. $\mathrm{CH}$ in cranial vault, are slow-growing lesions and typically occur in women in the second through fourth decades. Only less than $10 \%$ of cases are observed during the first decade as in our cases ${ }^{1-3,5,6,8-10)}$. The natural history of untreated lesions is poorly described. Cosmetic alterations, bleeding, and cranial nerves with progressive involvement in tumors of the skull base, as well as progressive exophthalmia and impairment of ocular movements in orbital lesions, were reported in some cases ${ }^{1,2,-10)}$. The symptoms are usually pain and visible or palpable bony hard mass or skull defect, slowly growing, covered by normal skin. Neurological deficits are unusual because these tumors tend to expand externally, but intracranial expansion has been reported ${ }^{8,9)}$. Radiography of the cranial vault is the most useful method to identify intraosseous $\mathrm{CH}$ as an intradiploic, well-circumscribed radiolucent rounded lesions with a partially sclerotic margins and sunburst or honeycomb pattern of trabeculations and protrusion into the external plate of skull. These characteristics are better defined on CT scan, which revealed an intradiploic hypodense lesion with rarefaction. The CT scan is more helpful than MRI in planning surgery because it shows the location and extent of tumor better in bone windows. The signal characteristics on MRI are variable. The lesion usually appears mottled and heterogeneous with both increased and decreased signal intensities on both T1- and T2-weighted images. The signal depends on the quantity of slow-moving venous blood and on the ratio of red marrow to converted fatty marrow present within the lesion. $\mathrm{CH}$ typically enhance after administration of gadolinium. High signal intensity on T2-weighted MRI may be caused by pooling of blood or slow-flowing blood. In angiographic examination, most of $\mathrm{CH}$ lacks a vascular blush, and diagnosis of hemangioma may not be suggested ${ }^{1-6,8-10)}$.

$\mathrm{CH}$ are composed of group of large, dilated blood vessels separated by fibrous tissue, whereas capillary hemangiomas lack fibrous septa and have smaller vascular lumens. Most of the $\mathrm{CH}$ in cranial vault are of the cavernous type, whereas vertebral $\mathrm{CH}$ are most frequently of the capillary type. They are supplied by branches of external carotid artery (middle meningeal artery and superficial temporal artery), arising in the cranial vaults. The bony trabecula seen within this tumor is believed to be the result of osteoclastic remodeling and osteoblastic reinforcement by the growing vascular tumor ${ }^{1-10)}$.

The differential diagnosis includes osteoma, osteoblastoma, aneurysmal bone cyst, giant cell tumor, fibrous dysplasia, sarcoma, meningioma, metastatic disease, Paget disease, dermoid and epidermoid cyst. These tumors are not difficult to identify by epidemiological, clinical and neuroimaging methods, but the frequent atypical or small cases are not so easy to differentiate $^{1-3,5,6,8-10)}$. Radiating lattice-like or web-like trabecular 
pattern of the skull by bony erosion may be seen in radiographs in cranial vault $\mathrm{CH}$. The osteoclastic activity of the tumor and following osteoblastic remodeling with trabecular bone result in the "sunburst" appearance on radiograph". Osteosarcoma can also present osteolytic lesion but it involves both inner and outer table. Histopathologic confirmation can be effective for making definite diagnosis.

The current treatment of choice for $\mathrm{CH}$ in cranial vault is surgical monobloc resection. To prevent recurrence, removal of the lesion plus a wide margin of uninvolved normal bone is always recommended. This method removes the tumor intact and remains the standard approach. Embolization of tumor before surgery is helpful in preventing excessive bleeding $^{1-3,5,6,8-10)}$. Recurrence of $\mathrm{CH}$ suggests that lesions develop from vasculature that is present already in response to a proliferative stimulus. Reconstruction of the cranial vault defect with methyl methacrylate often made favorable cosmetic results. The other therapeutic options for these tumors are curettage and radiation therapy ${ }^{1-3,5,6,8-10)}$. Curettage canal so involve excessive blood loss and disturbance of hemostasis, and carries a higher risk of recurrence than surgical excision. Radiation therapy may stop tumor growth but does not reduce the size of the tumor and has a risk of development of malignant transformation. The radiation therapy has a role in lesions that are felt to be unresectable or residual tumors from subtotal resections, such as those that deeply invade the bone, orbit, or cavernous sinus.

\section{CONFLICTS OF INTEREST}

No potential conflict of interest relevant to this article was reported.

\section{REFERENCES}

1. Khanam H, Lipper MH, Wolff CL, Lopes MB: Calvarial hemangiomas: report of two cases and review of the literature. Surg Neurol 55:63-67, 2001

2. Martínez-Lage JF, Torroba MA, Cuartero Pérez B, Almagro MJ, López López A, de la Rosa P: Cavernous hemangiomas of the cranial vault in infants: a case-based update. Childs Nerv Syst 26:861-865, 2010

3. Naama O, Gazzaz M, Akhaddar A, Belhachmi A, Asri A, Elmostarchid B, et al.: Cavernous hemangioma of the skull: 3 case reports. Surg Neurol 70:654-659, 2008

4. Nakayama Y, Tanaka A, Ueno Y, Yoshinaga S, Takano K: Scalp cavernous angioma presenting as sinus pericranii: diagnostic value of cerebral angiography and magnetic resonance imaging. Childs Nerv Syst 16:598-602, 2000

5. Nasi D, Somma L, Iacoangeli M, Liverotti V, Zizzi A, Dobran $\mathrm{M}$, et al.: Calvarial bone cavernous hemangioma with intradural invasion: An unusual aggressive course-Case report and literature review. Int J Surg Case Rep 22:79-82, 2016

6. Ramdasi R, Mahore A, Thorve S, Patil M: Intradiploic cavernous hemangioma presenting as multiple craters on skull. Acta Neurochir (Wien) 158:729-731, 2016

7. Silva RD, da Silva Cavalcante JE, Miranda EQ, Lopes DF, Souto LR: Giant hemangioma presenting as a scalp mass leading to a craniofacial deformity. J Maxillofac Oral Surg 12:218-223, 2013

8. Srinivasan VM, Karas PJ, Sen AN, Fridley JS, Gopinath SP: Intracranial hypertension caused by occipital calvarial hemangioma: Case report. World Neurosurg 91:672.e671-e673, 2016

9. Xu P, Lan S, Liang Y, Xiao Q: Multiple cavernous emangiomas of the skull with dural tail sign: a case report and literature review. BMC Neurol 13:155, 2013

10. Yang Y, Guan J, Ma W, Li Y, Xing B, Ren Z, et al.: Primary intraosseous cavernous hemangioma in the skull. Medicine (Baltimore) 95:e3069, 2016 